\title{
Down-regulation of acetolactate synthase compromises Ol-1- mediated resistance to powdery mildew in tomato
}

Dongli Gao ${ }^{1}$, Robin P Huibers ${ }^{1,2}$, Annelies EHM Loonen ${ }^{1}$, Richard GF Visser ${ }^{1}$, Anne-Marie A Wolters ${ }^{1}$ and Yuling Bai ${ }^{*}$

\begin{abstract}
Background: In a cDNA-AFLP analysis comparing transcript levels between powdery mildew (Oidium neolycopersici)susceptible tomato cultivar Moneymaker (MM) and near isogenic lines (NILs) carrying resistance gene Ol-1 or Ol-4, a transcript-derived fragment (TDF) M11E69-195 was found to be present in NIL-Ol-1 but absent in MM and NIL-Ol-4. This TDF shows homology to acetolactate synthase (ALS). ALS is a key enzyme in the biosynthesis of branched-chain amino acids valine, leucine and isoleucine, and it is also a target of commercial herbicides.

Results: Three ALS homologs ALS1, ALS2, ALS3 were identified in the tomato genome sequence. ALS1 and ALS2 show high similarity, whereas ALS3 is more divergent. Transient silencing of both ALS1 and ALS2 in NIL-Ol-1 by virus-induced gene silencing (VIGS) resulted in chlorotic leaf areas that showed increased susceptibility to O. neolycopersici (On). VIGS results were confirmed by stable transformation of NIL-Ol-1 using an RNAi construct targeting both ALS1 and ALS2. In contrast, silencing of the three ALS genes individually by RNAi constructs did not compromise the resistance of NIL-Ol-1. Application of the herbicide chlorsulfuron to NIL-Ol-1 mimicked the VIGS phenotype and caused loss of its resistance to On. Susceptible MM and On-resistant line NIL-Ol-4 carrying a nucleotide binding site and leucine rich repeat (NB-LRR) resistance gene were also treated with chlorsulfuron. Neither the susceptibility of MM nor the resistance of NIL-Ol-4 was affected.

Conclusions: ALS is neither involved in basal defense, nor in resistance conferred by NB-LRR type resistance genes. Instead, it is specifically involved in Ol-1-mediated resistance to tomato powdery mildew, suggesting that ALS-induced change in amino acid homeostasis is important for resistance conferred by $\mathrm{Ol}-1$.
\end{abstract}

Keywords: Acetolactate synthase, Oidium neolycopersici, Resistance, Solanum lycoperisum, Amino acid homeostasis

\section{Background}

In their natural environment plants are constantly attacked by a variety of pathogens. Nevertheless, plants can detect and evade most infection attempts through constitutive and inducible immune responses. The inducible responses consist of two layers [1]. The first layer is triggered by multifarious pathogen-associated molecular patterns (PAMPs). The perception of PAMPs by plant pattern recognition receptors (PRRs) stimulates a number of cellular events, which include production of

\footnotetext{
* Correspondence: Bai.Yuling@wur.nl

${ }^{1}$ Wageningen UR Plant Breeding, P.O. Box 386, 6700 AJ Wageningen, The Netherlands

Full list of author information is available at the end of the article
}

reactive oxygen species, activation of mitogen-activated kinases, enhanced expression of defense genes and production of antimicrobial compounds [2,3]. The second layer of inducible responses is activated by variable pathogen-specific effectors. Recognition of effectors by the plant is mostly mediated by a class of resistance proteins which contain nucleotide binding site and leucine rich repeat (NB-LRR) domains. The regulation and execution of both inducible responses involve hormone signalling pathways [4].

Emerging evidence illustrates that defense pathways are not only regulated by classical hormones, but also amino acid metabolic pathways constitute an important part of the plant immune system [5]. Besides the fact 
that some amino acids serve as precursors of antimicrobial compounds (e.g. glucosinolates) [6], amino acid homeostasis is pivotal for the outcome of plant-microbe interactions. A dominant nematode resistance gene in soybean encodes a serine hydroxymethyltransferase (SHMT), which plays a key role in one-carbon folate metabolism [7]. The SHMT allele in the resistant genotype encodes an isoform of the enzyme with altered kinetic properties compared with the isoform in susceptible genotypes. This altered SHMT enzyme is likely associated with perturbation of the folate pathway resulting in nutritional deficiency for nematodes. Overexpression of a pepper asparagine synthetase in Arabidopsis enhanced the resistance to bacterial and oomycete pathogens, which was correlated with increased asparagine levels [8]. Arabidopsis recessive downy mildew-resistant $(d m r 1)$ mutants defective in homoserine kinase were found to be resistant to the oomycete Hyaloperonospora arabidopsidis (Hpa). The resistance was homoserine-induced, and independent of known signalling pathways [9]. Suppression of the ortholog SIDMR1 in tomato resulted in elevated resistance to powdery mildew Oidium neolycopersici [10]. Resistance to Hpa was also obtained in Arabidopsis rar1-suppressor ( $r s p$ ) mutants, in which the level of threonine (Thr) was highly elevated [11]. The rsp1 mutant carries a mutation in the aspartate kinase 2 gene [11], which catalyzes the first step in the aspartate-derived amino acid pathway. The $r s p 2$ mutant contains a loss-of-function allele of dihydrodipicolinate synthase2 [11], which is the key enzyme in lysine biosynthesis. Disruption of an amino acid transporter LHT1 confers a broad spectrum disease resistance in Arabidopsis plants, likely as a result of deficiency of glutamine [12].

Oidium neolycopersici $(\mathrm{On})$ is an important biotrophic fungal disease for greenhouse crops. Unlike most powdery mildews that are host specific, $O n$ can infect a wide range of hosts, including species of the Solanaceae and Cucurbitaceae families [13]. A favourable strategy to control the disease consists of exploration of resistant alleles from wild species and introgression of these alleles into cultivated species to develop resistant cultivars. In tomato nine loci conferring resistance to $O n$ have been identified [14,15]. One of them - Ol-1 - originates from Solanum habrochaites G1.1560 [16], and confers incomplete resistance associated with slow hypersensitive response (HR) [17]. It is located on chromosome $6[16,18]$ and has been fine-mapped to a region encompassing six predicted genes, based on the sequence of tomato cultivar Heinz 1706 [19] \& unpublished results. None of the six genes encodes a protein with NB-LRR domains. Unravelling the identity of $\mathrm{Ol}-1$ has not been successful yet, because silencing of the predicted candidate genes individually did not attenuate the resistance level of the near-isogenic line carrying $\mathrm{Ol}-1$ (NIL-Ol-1) [unpublished results]. Another resistance gene - Ol-4 - which has been introgressed from S. peruvianum LA2172 confers complete resistance to $\mathrm{On}$ with fast HR [16]. It has been mapped to the $M i-1$ gene cluster on chromosome 6 [15]. Disease tests showed that NIL-Ol-4 was resistant to root-knot nematodes, indicating the presence of a functional $\mathrm{Mi}-1$ homolog encoding a NB-LRR type protein. Furthermore, silencing of $\mathrm{Mi}-1$ homologs in NIL-Ol-4 compromised the resistance to both $\mathrm{On}$ and root-knot nematodes, showing that $\mathrm{Ol}-4$ is a $\mathrm{Mi}-1$ homolog [20].

In a previous study designed to elucidate the pathways of $\mathrm{On}$ resistance, a cDNA-AFLP approach was used to identify transcript-derived fragments (TDF) showing differential presence or intensity in resistant tomato NILs relative to susceptible Moneymaker (MM) after mockinoculation or inoculation with powdery mildew $\mathrm{On}$ $[17,21]$. A BLAST analysis of the sequences of a number of differentially expressed TDFs was performed using the Sol Genomics Network (SGN) database to identify unigene sequences showing highest homology to each TDF. Subsequently, Tobacco Rattle Virus (TRV)-based Virus-Induced Gene Silencing (VIGS) constructs were generated targeting the unigenes. Then, VIGS was performed in the genotypes in which the TDF was detected to analyse whether silencing of targeted genes altered On resistance. In this way, it was shown that a putative glutathione $S$-transferase gene is required for Ol-1-mediated resistance against $O n$ [22].

In the present study we focused on another of these differentially expressed TDF (M11E69-195) and analysed its involvement in On resistance. M11E69-195 was specifically present in NIL-Ol-1 but absent in MM and NIL-Ol-4 [17,21]. The sequence of this TDF showed homology to acetolactate synthase (ALS). ALS (EC 2.2.1.6) is more frequently referred to as acetohydroxyacid synthase (AHAS) [23] in other studies. In this study, we describe it as ALS based on the annotation in the SGN database. ALS catalyzes the first step in the production of the branched-chain amino acids (BCAAs) valine, leucine and isoleucine [24]. It is extensively studied since it is a target of commercially successful herbicides. Different herbicide molecules can block substrate access to the active site of the ALS enzyme [25]. Here, we report the involvement of $A L S$ in $O l$-1-mediated resistance to powdery mildew in tomato.

\section{Results}

Down-regulation of two ALS genes simultaneously compromises Ol-1-mediated resistance to powdery mildew On

In the cDNA-AFLP study by Li et al. [17,21] TDF fragment M11E69-195 (No. 24 in Appendix 1 in [17]; No. 71 in Appendix 1 in [21]) was observed to be present in On-resistant NIL-Ol-1, but absent in On-susceptible $\mathrm{MM}$ and $\mathrm{On}$-resistant NIL-Ol-4. BLAST analysis of the 
sequence of this 195-bp TDF fragment was initially performed using the SGN database before the tomato genome sequence became publicly available. Highest homology was obtained for unigene SGN-U196237, a Capsicum annuum ALS gene (Additional file 1A). Primers were designed based on the U196237 sequence, and a 287-bp PCR product obtained using NIL-Ol-1 cDNA as template was cloned into VIGS vector TRV2, resulting in vector TRV-U196237 (Figure 1). Infiltration of TRVU196237 into NIL-Ol-1 induced morphological changes, including short stature and curled leaves with chlorotic areas (Figure 2A). Subsequently, VIGS plants were inoculated with On. Quantification of fungal biomass showed that there was a significant (3-fold) increase on plants infiltrated with TRV-U196237 compared to plants infiltrated with the empty vector (TRV-EV) (Figure 2B).

After the tomato genome sequence became accessible a new BLAST analysis of the sequence present in the VIGS vector was performed. This resulted in the identification of three putative $A L S$ genes in tomato named ALS1 (Solyc03g044330), ALS2 (Solyc07g061940) and ALS3 (Solyc06g059880) (Additional file 1B). The latter one, although present on chromosome 6 , does not reside in the Ol-1 region [19]. ALS1 and ALS2 predicted proteins are $94 \%$ identical at the amino acid level, while ALS3 is quite different from ALS1 and ALS2 (75\% and
78\% identity with ALS1 and ALS2, respectively) (Additional file 1C). The ALS1 and ALS2 genes are predicted to contain one exon, whereas $A L S 3$ is predicted to contain three exons (Figure 1). Alignment of the TDF sequence (derived from the NIL-Ol-1 line) with the three annotated $A L S$ genes showed that the TDF was probably derived from the ALS3 ortholog in S. habrochaites (Additional file 1A). However, alignment of the cloned fragment in the VIGS construct with the three annotated $A L S$ genes resulted in highest homology to ALS2. This discrepancy can be explained, because for construction of the VIGS vector primers were designed based on the SGN unigene showing highest homology to the TDF, but no unigene based on EST sequences from ALS3 was present in the SGN database. The alignment suggested that the VIGS vector targeted both $A L S 1$ and $A L S 2$, but not $A L S 3$, based on the assumption that an identical sequence of at least 21 nucleotides is necessary for efficient silencing. To validate the specificity of silencing, transcript levels of $A L S 1, A L S 2$ and $A L S 3$ in NIL-Ol-1 plants subjected to VIGS were measured by qRT-PCR using RNA isolated after pooling the third and fourth whole leaves of each plant. In this experimental set-up expression levels of the three $A L S$ genes were not significantly reduced in TRVU196237-infiltrated plants compared with TRV-EV-

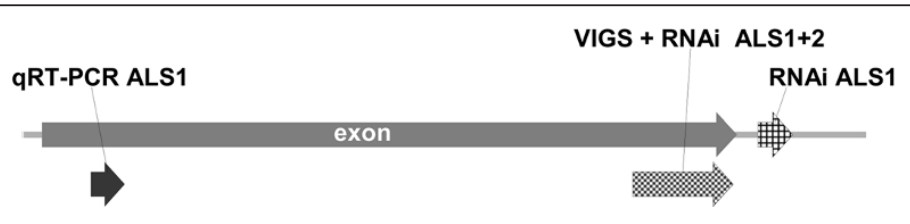

ALS1 genomic (2401 bp)

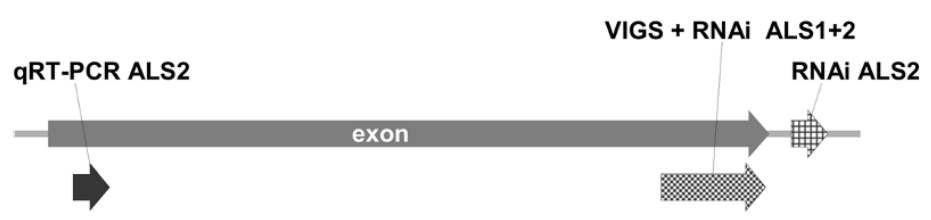

ALS2 genomic (2316 bp)

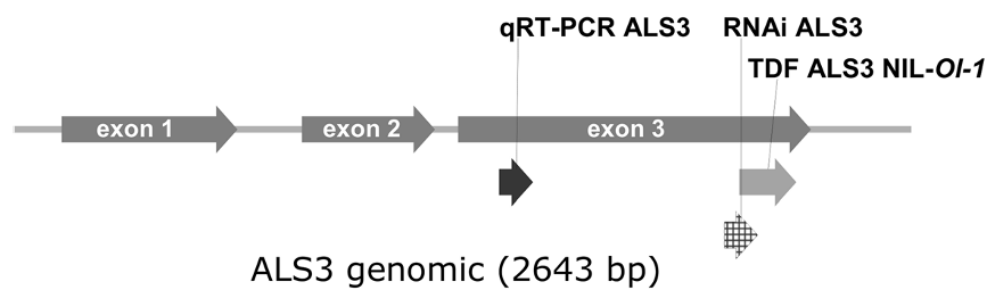

Figure 1 Schematic representation of the genomic sequences of tomato acetolactate synthase genes ALS1, ALS2 and ALS3. PCR fragments used in VIGS and RNAi constructs are indicated, as well as gene-specific fragments amplified in qRT-PCR analyses for quantification of gene expression. The fragment indicated as 'VIGS + RNAi ALS1 +2' was present in TRV-U196237 and also used for stable transformation using an RNAi construct. TDF M11E69-195 from NIL-Ol-1 showed the highest level of homology with exon 3 of ALS3. 

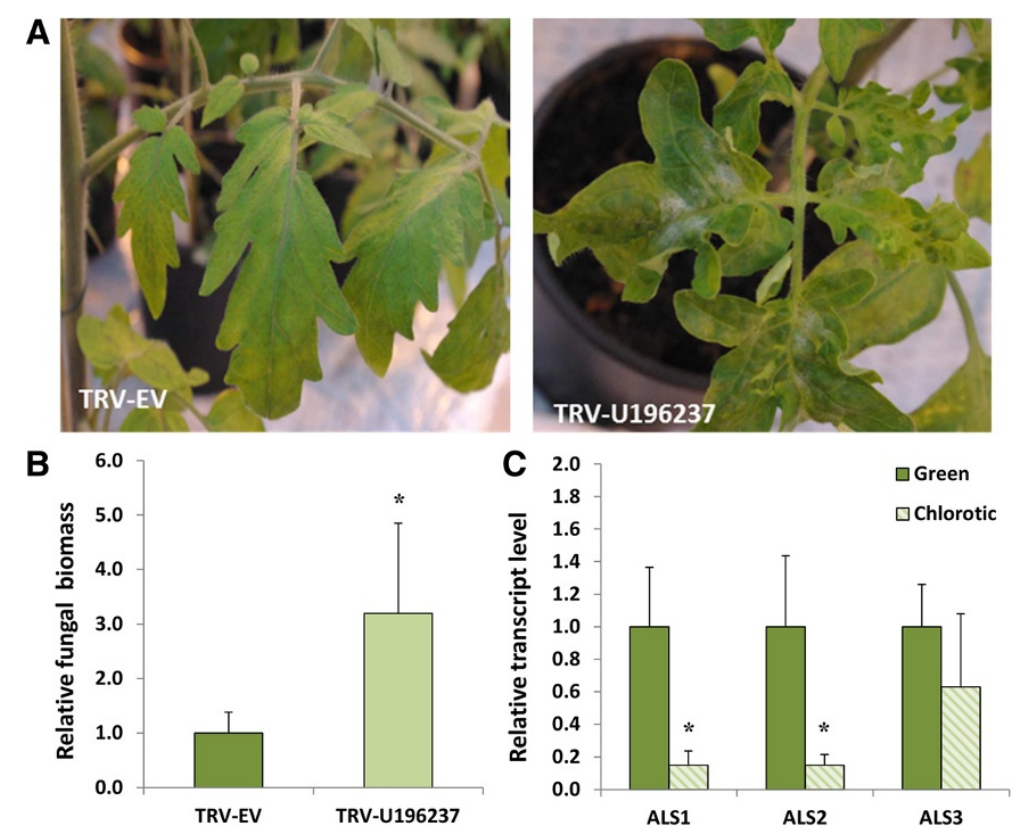

Figure 2 Down-regulation of two ALS genes simultaneously via VIGS compromises Ol-1-mediated resistance. (A), Effects of VIGS on growth and O. neolycopersici infection of NIL-Ol-1 plants infiltrated with empty vector as the control (TRV-EV) and TRV-U196237. (B), Quantification of fungal biomass of TRV-EV plants and TRV-U196237 plants. Values were normalized relative to elongation factor 1a (EF), and calibrated to levels in TRV-EV plants. Error bars represent standard deviation of five TRV-EV and ten TRV-U196237 plants. For each plant DNA was extracted from pooled $3^{\text {rd }}$ and $4^{\text {th }}$ leaves. (C), Transcript levels of ALS1, ALS2, and ALS3 in green and chlorotic areas of TRV-U196237 leaves. Values were normalized relative to $E F$, and calibrated to levels in green area. Error bars represent standard deviation of five biological replicates. Asterisks indicate significant difference from the control according to independent-samples t-test $(P<0.05)$.

infiltrated plants (data not shown), although the alteration in leaf morphology indicated a VIGS effect. However, we noticed that fungal colony growth was stronger on the chlorotic areas of the leaves than on the green areas. Therefore, transcript levels of ALS1, $A L S 2$ and $A L S 3$ were compared between leaf samples collected from excised green and chlorotic areas of TRV-U196237-infiltrated plants. The expression levels of $A L S 1$ and $A L S 2$ were significantly lower in chlorotic areas in comparison with green areas, while expression of ALS3 was also somewhat decreased in chlorotic areas, but not significantly (Figure $2 \mathrm{C}$ ). This indicated that the fragment present in the TRV-U196237 VIGS construct specifically silenced ALS1 and ALS2, but not ALS3.

To confirm the results obtained with VIGS, we generated stable transformants of NIL-Ol-1 in which both ALS1 and ALS2 were silenced by an RNAi construct (RNAi-ALS1 + 2) containing an inverted repeat of the same sequence as present in the TRV-U196237 vector. We expected that when $A L S 1$ and $A L S 2$ were efficiently silenced by RNAi the transformants would show the same visible phenotype as in VIGS, i.e. smaller plants with chlorotic leaves. Nine primary transformants (T1) were selfed to produce T2 families. For each T2 family ten plants were phenotypically examined. One T2 family (216) showed clear segregation for the phenotypic traits mentioned above (reduced stature and chlorotic leaves) (Figure 3A). The altered phenotype co-segregated with the presence of the silencing construct, as indicated by amplification of the $35 \mathrm{~S}$ promoter (Figure 3A). After inoculation with $\mathrm{On}$ the T2 plants with an altered phenotype showed increased sporulation when compared with the untransformed NIL-Ol-1 plants (Figure 3B), although full susceptibility as in cultivar MM was not reached. Fungal growth and transcript levels of $A L S$ genes were quantified in NIL-Ol-1 plants and in T2 plants with altered phenotype. The results showed that the $O n$ fungal biomass in these T2 plants was significantly increased compared with NIL-Ol-1 (Figure 3C). As expected, the gene expression levels of both ALS1 and $A L S 2$ were significantly suppressed in the RNAiALS1 + 2 T2 plants, whereas ALS3 expression was not significantly reduced (Figure $3 \mathrm{D}$ ).

In addition to the production of stable transformants in which both $A L S 1$ and $A L S 2$ were silenced simultaneously, stable NIL-Ol-1 transformants were produced in which the three $A L S$ genes were silenced individually to evaluate their involvement in $\mathrm{Ol}-1$ resistance. No crosssilencing was observed (Additional file 2). Transformed $\mathrm{T} 1$ plants were selfed to obtain T2 families. One T2 family for $A L S 1$, three for $A L S 2$, and two for $A L S 3$ were obtained. The NPTII-containing, and thus transgenic, T2 


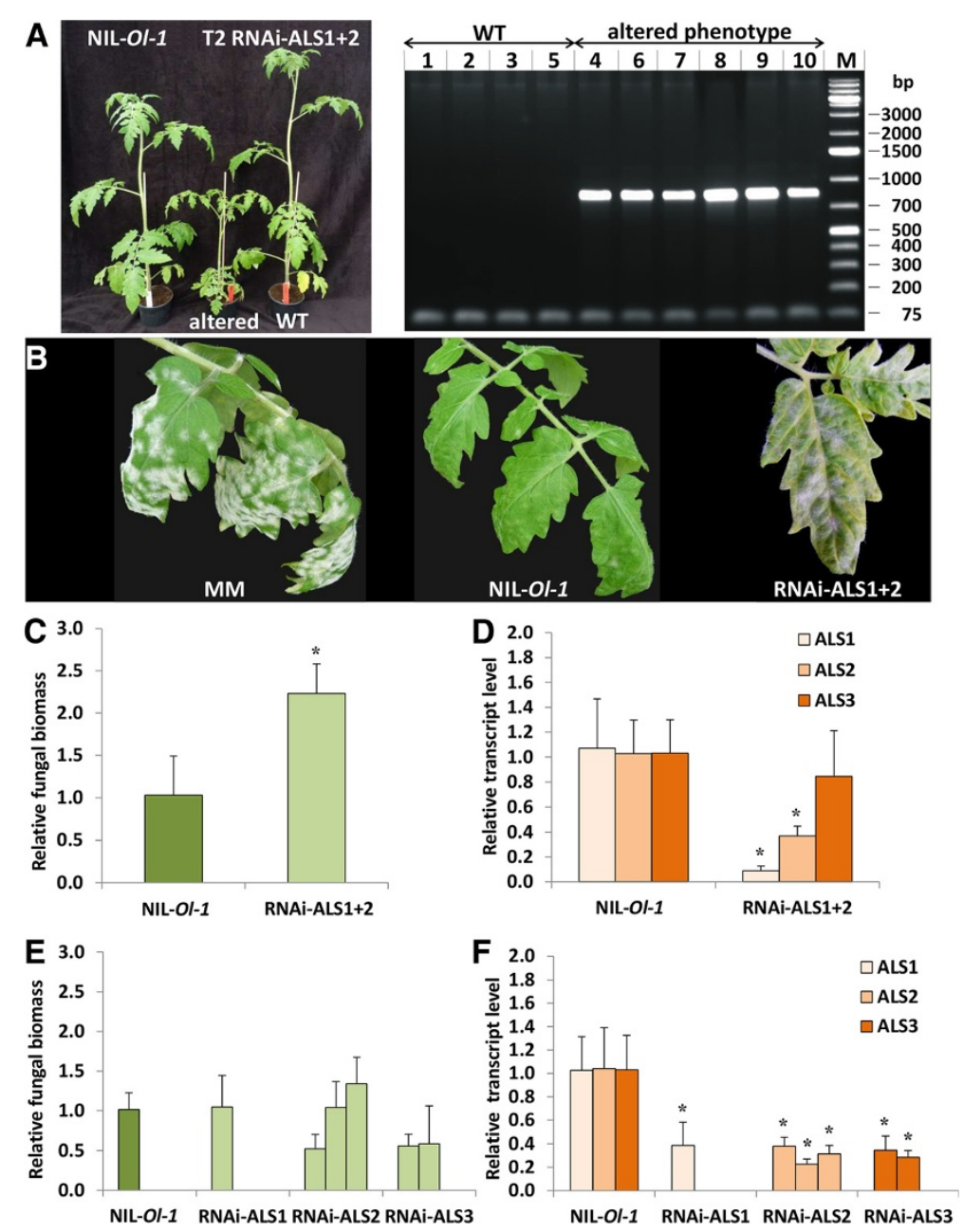

Figure 3 Assessment of resistance to Oidium neolycopersici (On) in RNAi transformants of NIL-OI-1. (A), Segregation for phenotypic traits in T2 plants obtained after selfing RNAi-ALS1 + 2 transformant 216, compared with the NIL-Ol-1 untransformed plant. PCR analysis of the $35 S$ promoter of the silencing construct showed co-segregation of altered phenotype with presence of the expected PCR product. (B) On symptoms on MM, NIL-Ol-1 and NIL-Ol-1 transformant obtained with the ALS1 + 2 RNAi construct (T2 plant). (C), On fungal biomass and (D), transcript levels of ALS1, ALS2, and ALS3 in RNAi-ALS1 + 2 T2 plants of family 216 showing an altered phenotype, compared with that in NIL-Ol-1 plants. (E), On fungal biomass and (F), transcript levels of ALS1, ALS2, and ALS3 in NPTII-containing T2 plants in which three ALS genes were targeted individually by RNAi (RNAi-ALS1, RNAi-ALS2, and RNAi-ALS3). For each plant RNA was isolated from pooled $3^{\text {rd }}$ and $4^{\text {th }}$ leaves. Values were normalized relative to $\mathrm{EF}$, and calibrated to the levels in untransformed NIL-Ol-1 plants. Error bars represent standard deviation of three biological replicates. Asterisks indicate significant difference from the controls according to independent-samples t-test and one way analysis of variance $(P<0.05)$.

plants were selected by PCR analysis. The transgenic T2 progeny showed a significant reduction of expression of the targeted $A L S$ gene (Figure 3F). Silencing of the three $A L S$ genes individually did not lead to morphological alteration, and fungal abundance was not enhanced compared to that in untransformed NIL-Ol-1 plants (Figure 3E). The fact that suppression of individual $A L S$ genes did not compromise $\mathrm{Ol}$-1-mediated resistance, but suppression of at least two $A L S$ genes compromised the resistance indicated that the function of $A L S$ genes is likely overlapping.

We did not try to generate a construct targeting all three genes simultaneously, because no continuous stretch of at least 21 identical nucleotides is present when aligning the complete coding sequences of the three $A L S$ genes (Additional file 1B).

\section{ALS is specifically involved in Ol-1-mediated resistance}

Acetolactate synthase is a well-known target for commercial herbicides, which block binding of substrates to the active site of the ALS enzyme [25]. We employed this system to determine whether ALS was generally involved in powdery mildew resistance or specifically involved in Ol-1-mediated resistance. The herbicide chlorsulfuron was used as the ALS inhibitor. First, we studied the effect of herbicide treatment in NIL-Ol-1 
plants. As chlorsulfuron was dissolved in acetone, we included plants to which only acetone was applied as well as plants to which water was applied as controls. The herbicide application caused inhibition of shoot growth and overall chlorosis of the plant (Figure 4A). Quantification of fungal DNA showed that a significant increase of fungal biomass was attributable to herbicide treatment, as compared with the acetone control (Figure 4A and $\mathrm{B})$.

As powdery mildew fungi depend on living tissue for nutrient uptake, we wondered whether the probable perturbation of amino acid homeostasis due to silencing of $A L S$ could be exploited by the pathogen, and in turn influence the basal defense. To address this question we treated susceptible tomato MM with chlorsulfuron. If ALS is important for basal defence against powdery mildew, one would anticipate an increase of sporulation. After herbicide treatment we observed morphological changes in MM plants, which were similar to those in NILOL-1 plants. However, fungal biomass in chlorsulfurontreated MM plants was similar to fungal biomass in water- and acetone-treated MM, suggesting that ALS was not involved in basal defence (Figure 4B). Chlorsulfuron was also applied to NIL-Ol-4 plants to determine whether ALS is generally involved in powdery mildew resistance signalling pathways. Quantification of fungal biomass showed that herbicide-treated NIL-Ol-4 plants retained a similar resistance level to powdery mildew as the control NIL-Ol-4 plants, suggesting that ALS is dispensable for resistance conferred by $\mathrm{Ol}-4$, encoding a NB-LRR type protein (Figure 4B).

Similar to the results obtained with the VIGS and RNAi plants, we observed that, although NIL-Ol-1 plants in which ALS function was impaired showed increased susceptibility to $\mathrm{On}$, full susceptibility as in cultivar MM was not reached. This is shown in Figure $4 C$, in which fungal biomass in NIL-Ol-1 and NIL-Ol-4 plants is calibrated to the level in water-treated MM.

\section{Expression of $A L S$ genes upon powdery mildew attack in NIL-OI-1 and MM}

To investigate the response of three $A L S$ genes under powdery mildew attack, their transcript levels were measured in NIL-Ol-1 and MM. Expression of ALS1 and $A L S 2$ was detected in both genotypes, and they were not induced by powdery mildew inoculation in either genotype (Figure 5). ANOVA analysis indicated that there was no significant difference in expression levels of
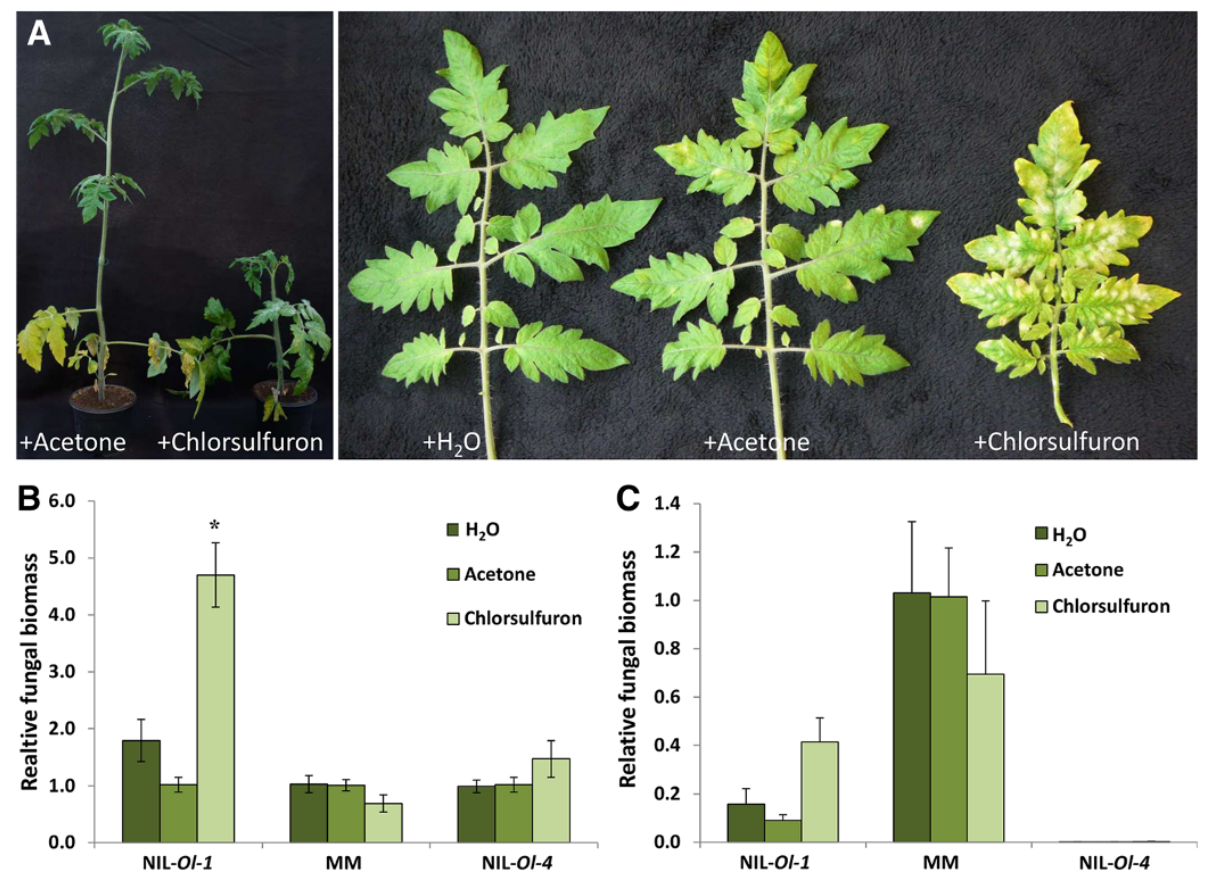

Figure 4 ALS is specifically involved in Ol-1-mediated resistance against Oidium neolycopersici (On). (A), Phenotype of NIL-Ol-1 plants in soil to which acetone (+ Acetone) or chlorsulfuron dissolved in acetone (+ Chlorsulfuron) has been added, and close-up of On development on the leaves. (B), Relative On fungal biomass on NIL-Ol-1, Moneymaker (MM) and NIL-Ol-4 plants grown in soil to which water $\left(\mathrm{H}_{2} \mathrm{O}\right)$, acetone or chlorsulfuron has been added. For each plant DNA was extracted from pooled $3^{\text {rd }}$ and $4^{\text {th }}$ leaves. Values are normalized relative to $E F$, and calibrated to level on plants grown in soil with acetone. Error bars represent three biological replicates for $\mathrm{H}_{2} \mathrm{O}$ and acetone treatments respectively, and 5 or more replicates for chlorsulfuron treatment. Two independent experiments were performed with similar results, and data from one experiment are presented. Asterisk indicates significant difference from the controls according to one way analysis of variance $(P<0.05)$. (C), Relative On fungal biomass on NIL-OI-1, MM and NIL-Ol-4 plants as in Panel B, but calibrated to the level on water-treated MM plants. 

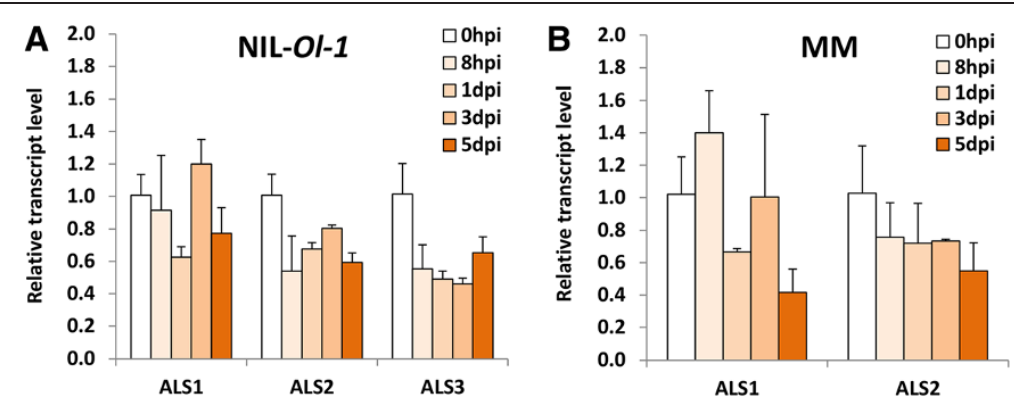

Figure 5 Expression profiles of three ALS genes in tomatoes upon powdery mildew attack. (A) NIL-Ol-1 and (B) Moneymaker (MM). Samples were harvested at 0 hpi (prior to inoculation), 8 hpi (hours post inoculation), 1 dpi (days post inoculation), 3 dpi and 5 dpi. For each biological replicate the 3rd and 4th leaves were pooled from three plants. Values are normalized relative to $E F$, and expression level at each time point after inoculation were calibrated to levels of counterpart plants without inoculation. Error bars represent standard deviation of three biological replicates. To test whether expressions of ALS1 and ALS2 were different between NL-OI-1 and MM, two-way between groups ANOVA was used.

ALS1 and ALS2 between NIL-Ol-1 and MM. ALS3 expression was only detected in NIL-Ol-1, while in MM it may be either weakly expressed below detection level, or not expressed at all (Figure 5). We could exclude the possibility that primers for quantifying ALS3 expression were not suitable for MM because a PCR product of the expected size was obtained using genomic DNA as template. Further, RNA-seq data of ALS3 (Solyc06g059880) from tomato cultivar Heinz [26] confirm that ALS3 is not expressed in tomato leaves (Additional file 3).

\section{Discussion}

In a screen of differentially expressed transcripts showing a difference in presence or intensity when comparing powdery mildew-resistant NILs with susceptible MM, TDF M11E69-195 was identified [17,21], which shows homology to acetolactate synthase. This TDF was specifically present in NIL-Ol-1, but absent in both MM and NIL-Ol-4 $[17,21]$. By targeting acetolactate synthases via VIGS, RNAi and herbicide application, we demonstrated that ALS activity was specifically important for Ol-1based resistance (Figures 2B, 3C and 4B). ALS does not seem to be involved in basal defense as indicated by unchanged susceptibility after herbicide treatment of MM, nor required for resistance controlled by NB-LRR-type resistance genes as indicated by the results from herbicide treatment of NIL-Ol-4 (Figure 4B).

\section{Possible involvement of $A L S 3$ in Ol-1-mediated resistance to On}

The fact that TDF M11E69-195 was observed in NILOl-1, but absent in both MM and NIL-Ol-4 could be caused solely by the presence of nucleotide polymorphisms between MM and NIL-Ol-1. However, we have shown that the corresponding ALS3 gene is truly differentially expressed, as ALS3 transcripts were observed in leaves from NIL-Ol-1 but not in MM leaves (Figure 5). The sequence of M11E69-195 from NIL-Ol-1 showed higher similarity to $A L S 3$ than to $A L S 1$ and $A L S 2$ from $S$. lycopersicum (Additional file 1A). ALS3 (Solyc06g059880) is located on the long arm of chromosome 6, but not in the Ol-1 region. ALS1 and ALS2 are located on chromosomes 3 and 7, respectively. As NIL-Ol-1 only contains (part of) chromosome 6 of S. habrochaites G1.1560 whereas all other chromosomes are from S. lycopersicum $\mathrm{MM}$, we expected that the ALS1 and ALS2 genes from NIL-Ol-1 were identical to those from MM. This was indeed observed after sequencing complete $A L S 1$ and $A L S 2$ cDNAs from NIL-Ol-1 (data not shown). In contrast, sequencing of the complete ALS3 cDNA from NIL-Ol-1 revealed a number of SNPs and indels in NIL-Ol-1 compared to the predicted sequence from tomato cultivar Heinz in the SGN database (Additional file 1B). NIL-Ol-4, containing an introgression of part of chromosome 6 from S. peruvianum accession LA2172 [19], was shown to contain ALS1 and ALS2 sequences identical to those from $\mathrm{MM}$, whereas the ALS3 sequence from NIL-Ol-4 differed from both MM and NIL-Ol-1.

The VIGS and RNAi constructs targeted ALS1 and ALS2, but not ALS3 (Figures 2C and 3D). As ALS1 and ALS2 in all three genotypes are identical, but the effect of silencing is specific for NIL-Ol-1, we wonder whether ALS3 plays a role in resistance to tomato powdery mildew conferred by Ol-1. Although ALS3 is homologous to acetolactate synthase genes whose function has been proven, the exact function of the ALS3 protein is unknown. In plants ALS is a heteromultimer, consisting of catalytic and regulatory subunits $[25,27,28]$. All three tomato proteins ALS1, ALS2 and ALS3 are homologous to known catalytic subunits, such as the SuRA and SuRB proteins of Nicotiana tabacum [29]. In Solanaceous species from which genome sequences are available three $A L S$ genes coding for catalytic subunits are present. In contrast, Arabidopsis only contains one $A L S$ gene encoding the catalytic subunit, i.e. At3g48560.

In $\mathrm{MM}$ and NIL-Ol-4 only ALS1 and ALS2 are expressed in leaves, while $A L S 3$ is not. Similarly, the 
orthologs of ALS3 in S. pimpinellifolium and S. tuberosum are not expressed in leaves (Additional file 3). NIL$\mathrm{Ol}-1$ is exceptional, as in this genotype ALS3 is expressed in leaves, together with ALS1 and ALS2, and therefore ALS3 may be incorporated in the ALS holoenzyme. Possibly, the presence of different catalytic subunits in the ALS holoenzyme confers different functionalities or substrate specificities. Although silencing of only ALS3 in the NIL-Ol-1 background did not result in increased susceptibility to $\mathrm{On}$ (Figure 3E), this does not exclude the possibility that ALS3 is involved in resistance. The obtained transformants showed significant silencing of $A L S 3$ (Figure 3F), but no complete silencing comparable with a knock-out mutation was achieved. Additional experiments are needed to elucidate the function of $A L S 3$ in leaves of NIL-Ol-1, for example expression of the $S$. habrochaites ALS3 gene in MM background.

\section{Involvement of amino acid homeostasis caused by altered ALS activity in Ol-1-mediated resistance}

Although acetolactate synthase is a known target of several herbicides, it is unclear how herbicide-binding affects the amino acid metabolism in plants. Scheel and Casida [30] found that chlorsulfuron treatment of soybean suspension cultures caused a decrease of the valine and leucine contents, but had no effect on other amino acids. They showed that growth inhibition by chlorsulfuron was alleviated by supplying exogenous valine or leucine, or a combination of valine, leucine and isoleucine. Consistent with a reduction of BCAAs caused by an ALS-affecting herbicide, Ray [31] observed that addition of valine and isoleucine to excised pea root cultures reversed herbicide-induced growth inhibition. Growth retardation can also result from ALS feedback inhibition by individual end products. Chen et al. [28] showed that addition of valine or leucine to the growth medium inhibited root growth of Arabidopsis seedlings, whereas addition of isoleucine had no effect. When a combination of valine + isoleucine, or leucine + isoleucine was added to the medium root growth inhibition was less pronounced, suggesting isoleucine counteracted the inhibitory effect of valine and leucine [27]. Royuela et al. [32] detected an increase in the relative proportion of some amino acids other than BCAAs in chlorsulfurontreated wheat and maize. Höfgen et al. [33] silenced $A L S$ in potato by antisense inhibition, resulting in a decrease of ALS activity of up to $85 \%$. Strong silencing of $A L S$ resulted in severe growth retardation and stunting, and leaf chlorosis. Similar phenotypic alterations were obtained by treatment with an imidazolinone herbicide. Measurement of amino acids showed an accumulation of total free amino acids as well as perturbed composition in antisense and herbicide-treated plants. Unexpectedly, instead of decreased levels, elevated amounts of amino acids including valine, leucine and isoleucine were observed, especially in older sink leaves.

Recently, another example of a link between herbicide resistance, increased amino acid levels, and resistance to fungal species was reported. Patent US8383887 [34] discloses that corn plants expressing the bacterial $g d h A$ gene (NADPH-dependent glutamate dehydrogenase) are resistant to aflatoxin accumulation following Aspergillus infection. Furthermore, corn and tobacco plants transformed with the $g d h A$ gene are resistant to root rot following Fusarium virguliforme infection. Previously, it has been shown that tobacco plants transformed with the $g d h A$ gene show an increased level of resistance to the herbicide glufosinate [35] and that total free amino acids were increased in these plants [36,37].

Taken together, the effect of ALS inhibition on levels of individual amino acids is difficult to predict, as it seems to depend on the level of residual ALS activity in different tissues, and the feedback-inhibition effect of (combinations) of individual amino acids. Despite this, we investigated whether BCAAs contents influence powdery mildew susceptibility or resistance in tomato cultivar MM and NIL-Ol-1 by exogenously applying leucine, isoleucine and valine [10] \& Additional file 4. Homoserine and threonine were also included in the experiment, because they were found to affect plant immunity, and threonine is the precursor of isoleucine. If a higher level of BCAAs contributes to $\mathrm{Ol}$-1-mediated resistance, we expected to gain powdery mildew resistance to some degree in MM with elevated BCAAs levels. Quantification of fungal DNA showed that only exogenous application of homoserine significantly reduced the susceptibility of $\mathrm{MM}$ and increased resistance of NIL-Ol-1 to $\mathrm{On}$, whereas application of the other amino acids did not alter the responses of $\mathrm{MM}$ and NIL-Ol-1 to On [10] \& Additional file 4. We also did not observe the growth retardation which can be caused by individual end products, possibly because the concentration was not sufficiently high to cause this. The results suggested that instead of an elevated level, a reduced level of BCAAs or changed compositions of individual amino acids may play a role in $\mathrm{O}$-1-mediated powdery mildew resistance. Amino acid deprivation is known to activate defences in Arabidopsis. For instance, the accumulation of camalexin, a pathogen-inducible antimicrobial phytoalexin was induced by BCAAs starvation [38]. An alternative hypothesis is the involvement of an amino acid-derived signal(s) in defense signalling pathways, as suggested for Arabidopsis genes ALD1 and AGD2 encoding aminotransferases [39]. Furthermore, studies on plant hormone conjugates showed that jasmonate (JA) can conjugate BCAAs [40] and, in particular, JA-isoleucine is the main bioactive form of the hormone [41]. In addition, altered expression of an 
enzyme involved in conjugation affects salicylic acidmediated disease resistance [42].

In the case of $\mathrm{Ol}-1$, perturbation of amino acid balance by silencing of $A L S$ or herbicide treatment may impair the integrity of the signalling network, leading to the loss of resistance conferred by $\mathrm{Ol}-1$. The unknown identity of $\mathrm{Ol}-1$ makes it harder to understand the link between Ol-1-mediated resistance and amino acid homeostasis. Cloning of $\mathrm{Ol}-1$, determination of amino acid homeostasis, and dissection of changes in hormone signalling pathways will aid in understanding the requirement of ALS activity for Ol-1-based resistance and shed light on the interaction of amino acid metabolism and plant immunity.

\section{Conclusion}

Tomato genome encodes three $A L S$ genes. Silencing of each of them did not attenuate $\mathrm{Ol}$-1-mediated resistance to tomato powdery mildew, while down-regulation of both $A L S 1$ and $A L S 2$ simultaneously or inhibition of the ALS activity resulted in the loss of $\mathrm{Ol}-1$-mediated resistance to tomato powdery mildew. Further research on cloning of $\mathrm{Ol}-1$ and association of amino-acid homeostasis with ALS activity may provide insight into the role of amino acid metabolism in tomato resistance to powdery mildew.

\section{Methods}

\section{Plant materials, fungal isolate and inoculation}

All the near isogenic lines (NIL) have been described previously [15]. They were obtained by crossing wild tomato species containing the resistance gene(s) with $S$. lycopersicum cultivar Moneymaker (MM), three backcrosses with MM, followed by two selfings (BC3S2 plants). Ol-1 was introgressed from $S$. habrochaites G1.1560, while Ol-4 was introgressed from S. peruvianum (or S. arcanum) LA2172. Oidium neolycopersici $(\mathrm{On})$ isolate Netherlands was maintained on susceptible MM plants in a growth chamber at $21 / 19^{\circ} \mathrm{C}$ (day/night). Fungal spores were washed off from heavily infected tomato leaves with tap water and diluted to a concentration of $2.5 \times 10^{4}$ spores per $\mathrm{mL}$. The inoculum was evenly sprayed on 3 to 4 weeks-old plants.

\section{Virus-induced gene silencing (VIGS)}

VIGS was performed using the TRV-based vector system [43]. Primers for the TRV2 construct targeting SGNU196237 were Fw-U196237-caccCAATGGGAGGATCG GTTCTA and Rv-U196237-ATCTCCCATCACCCTCT GT. A 290-bp fragment was amplified from cDNA of NIL-Ol-1 plants, and subsequently cloned into pENTR/DTOPO vector (Invitrogen). After verification of the sequence the fragment was introduced into the pTRV2attR1-attR2 vector [43] via $L R$ recombination. The resulting TRV-U196237 vector was transformed into Agrobacterium strain GV3101. To establish VIGS, cotyledons of 10-days-old plants were agroinfiltrated with a mixture of TRV1 and TRV-U196237 (combined in a 1: 1 ratio). As a control, a mixture of TRV1 and empty TRV2 vector (TRV-EV) was used. Three weeks after agroinfiltration the plants were sprayed with $O n$ inoculum. Three independent experiments were performed. For two experiments disease symptoms were visually scored 21 dpi by counting the number of fungal colonies on the leaves. For one experiment silencing level and fungal growth were quantified by qRT-PCR, using RNA from five plants for TRV-EV and 10 plants for TRV-U196237.

\section{Generation of stable silenced lines}

To suppress both $A L S 1$ and $A L S 2$ by RNAi, the same fragment as used in VIGS construct TRV-U196237 was introduced into the pHellsgate8 vector [44]. For the purpose of targeting ALS1 and ALS2 separately, primers were designed based on the 3' UTR sequences. For ALS1: FwALS1-caccGCCAAAAGTGTTCGATTTGT and Rv-ALS1AGTGAACATAAATACCAAGTAGAAGAT. For ALS2: Fw-ALS2-caccTGTTTACTTAAAAGTTTTTC ATTGTG and Rv-ALS2-TTAGTCATACTAAATAGAGCTCCAAA. To suppress $A L S 3$, primers were designed based on the sequences in coding region: Fw-ALS3-caccTTATCTTGGAAATCCTTCTAACAA and Rv-ALS3-TTCTTATGA ATCACTTGAGCA. Fragments amplified with abovementioned primers were introduced into pHellsgate8 vector [40] and finally transformed into Agrobacterium strain AGL1 + virG. For generation of silenced lines the protocol described by Huibers et al. [10] was used. Primary transformants (T1) were selfed to produce T2 families. For each segregating T2 family, CaMV $35 \mathrm{~S}$ promoter primers XhoFw-TGCTGACCCACAGATGGTTA and 35S2-GATAGT GGGATTGTGCGTCA [45] amplifying a 756-bp fragment, or NPTII primers Fw-NPTII-TTCCCCTCGGTATCCA ATTA and Rv-NPTII-GATTGTCTGTTGTGCCCAGT amplifying a 170-bp fragment from the pHellsgate8 $\mathrm{T}$ DNA, were used to select transgenic T2 plants.

\section{Herbicide application}

Chlorsulfuron was purchased from Aldrich-Sigma (PS1065), and the powder was dissolved in acetone $(0.2 \mathrm{mg} / \mathrm{ml})$. The herbicide solution was applied to $30-$ day-old plants of MM and NIL-Ol-1 growing in ø14 $\mathrm{cm}$ pots in normal potting soil. Before application, watering was suspended for two days to ensure that the solution could be absorbed completely. As controls, water and acetone were applied. The chlorsulfuron solution, acetone and water were added to the soil with a pipette $(8 \mathrm{ml}$ per pot). After this, the plants were challenged with powdery mildew $\mathrm{On}$ at the same day of herbicide application. Per genotype 15 plants 
were treated with chlorsulfuron, and five plants with water or acetone.

\section{Quantitative reverse transcription PCR (qRT-PCR) and data analysis}

For quantification of fungal biomass, DNA or RNA extracted from tomato leaves was used. For quantification of transcript levels, RNA was used. DNA was isolated with DNeasy plant mini kit (Qiagen). Total RNA was extracted from leaflets using the RNeasy kit (Qiagen). After removal of DNA with DNase I (Invitrogen), $1 \mu \mathrm{g}$ total RNA was used for cDNA synthesis using SuperScript II Reverse Transcriptase kit (Invitrogen). Quantitative realtime PCR was conducted using the iQ SYBR Green supermix (Bio-Rad) and the CFX96 Real-Time system (Bio-Rad). The PCR amplification consisted of an initial denaturation step of $3 \mathrm{~min}$ at $95^{\circ} \mathrm{C}$, followed by denaturation for $15 \mathrm{sec}$ at $95^{\circ} \mathrm{C}$, annealing and extension for $1 \mathrm{~min}$ at $60^{\circ} \mathrm{C}$ for 39 cycles, then a final melt step from $65^{\circ} \mathrm{C}$ to $95^{\circ} \mathrm{C}$ ramp with $0.5^{\circ} \mathrm{C}$ increments per cycle to monitor specificity. Primers used for fungal quantification were $\mathrm{Fw}-\mathrm{On}$-CGCCAAAGACCTAACCAAAA and Rv-On-AGCCAAGAGATCCGTTGTTG. Primers for tomato elongation factor $1 \alpha(E F)$ were Fw-EF-GGAACTTGAGAAGGAGCCTAAG and Rv-EF-CAACACCAACA GCAACAGTCT [46]. For detection of relative transcript levels of the $A L S$ genes primers were Fw-ALS1-CGCTCAACATAATCGTCGTG and Rv-ALS1-ACGGGAAACGAAT GTTTCAG for ALS1; Fw-ALS2-CCCTTCTTCCCAAATCTACCT and Rv-ALS2-TTGAAACAGTGAAACGGCTATG for ALS2; Fw-ALS3-TTTGCTGCTAGCATTTGGAG and Rv-ALS3- GGAGTCGATATCAATGTGAACAA for ALS3. For the time-course experiment in which the expression of three $A L S$ genes was monitored after inoculation with On, the same set of primers was used as for detection of relative transcript levels of each $A L S$ gene after silencing. For analysis of the relative expression level and fungal biomass the $2^{-\Delta \Delta C t}$ method as described by Livak and Schmittgen [47] was used. Data were statistically examined using independent-samples t-test, one-way analysis of variance or two-way between groups ANOVA based on Post-hoc comparisons using Tukey's HSD test $(P<0.05)$. All analyses were performed using SPSS Statistics 20 following the instructions of SPSS Survival Manual $4^{\text {th }}$ edition [48].

\section{Additional files}

Additional file 1: Sequence alignments. (A), Sequence alignment of TDF M11E69-195 and ALS PCR fragment used in VIGS and RNAi constructs $\mathrm{ALS} 1+2$ (both from NIL-Ol-1) with the corresponding part of unigene SGN-U196237 from Capsicum annuum, and of ALS1, ALS2 and ALS3 transcripts from tomato cultivar Heinz. Nucleotides identical with the ALS3 sequence are highlighted. (B), Sequence alignment of ALS1, ALS2 and ALS3 coding sequences (CDS) of tomato cultivar Heinz, and the ALS3 CDS of
NIL-Ol-1. Nucleotides identical with the Heinz ALS3 sequence are highlighted. (C), Sequence alignment of protein sequences from tomato cultivar Heinz ALS1, ALS2 and ALS3 with ALS3 from NIL-Ol-1. Amino acids identical with the ALS3 sequence are highlighted.

Additional file 2: Absence of cross-silencing by RNAi constructs targeting individual ALS genes. Cross-silencing was not detected in three representative silenced lines in each of which a specific $A L S$ gene was targeted by RNAi (RNAi-ALS1, 2 and 3). Values were normalized relative to $E F$, and calibrated to the levels in untransformed NIL-Ol-1 plants. Error bars represent standard deviation of three biological replicates.

Additional file 3: RNA-seq data of $A L S 1, A L S 2$ and $A L S 3$ genes. Expression level of ALS1, ALS2 and ALS3 genes from tomato, $S$. pimpinellifolium and potato in leaves and root (for tomato) or tuber (for potato) derived from RNA-seq data, and indicated as Fragments per Kilobase of exon per Million fragments mapped (FPKM) or Reads per Kilobase of transcripts per Million mapped (RPKM) values.

Additional file 4: Effect of exogenous application of amino acids on Oidium neolycopersici (On) fungal growth. Quantification of On fungal biomass, 8 days post inoculation of NIL-Ol-1 plants sprayed with different amino acids solutions. Amino acids homoserine (HS), threonine (Thr), or branched-chain amino acids valine (Val), isoleucine (lle) or leucine (Leu) were applied as described in Huibers et al. [10]. Data indicate the mean of three biological replicates with error bars representing the standard deviation. The asterisk indicates significant difference from the $\mathrm{H}_{2} \mathrm{O}$ control according to one way analysis of variance $(P<0.05)$.

\section{Competing interests}

The authors declare that they have no competing interests.

\section{Authors' contributions}

DG carried out the experiments. AEHML participated in the herbicide experiment. DG, RPH, and YB designed the experiments. YB drafted the outline of the manuscript, and DG and AMAW wrote the paper. YB and RGFV commented on the manuscript. All authors read and approved the final manuscript.

\section{Acknowledgments}

Dongli Gao was supported by the China Scholarship Council (CSC).

\section{Author details}

${ }^{1}$ Wageningen UR Plant Breeding, P.O. Box 386, 6700 AJ Wageningen, The Netherlands. ${ }^{2}$ Present address: Enza Zaden Beheer B.V., Haling 1E, 1602 DB Enkhuizen, The Netherlands.

Received: 9 October 2013 Accepted: 9 January 2014

Published: 17 January 2014

\section{References}

1. Jones JDG, Dangl JL: The plant immune system. Nature 2006, 444(7117):323

2. Schwessinger B, Zipfel C: News from the frontline: recent insights into PAMP-triggered immunity in plants. Curr Opin Plant Biol 2008, 11(4):389.

3. Stotz $\mathrm{H}$, Waller $F$, Wang $\mathrm{K}$ : Innate immunity in plants: the role of antimicrobial peptides. In Antimicrobial Peptides and Innate Immunity, Progress in inflammation research. Edited by Hiemstra PS, Zaat SAJ. Springer Basel; 2013:29-51.

4. Bari R, Jones JDG: Role of plant hormones in plant defence responses. Plant Mol Biol 2009, 69(4):473.

5. Zeier J: New insights into the regulation of plant immunity by amino acid metabolic pathways. Plant Cell and Environment 2013. doi:10.1111/ pce.12122.

6. Sønderby IE, Geu-Flores F, Halkier BA: Biosynthesis of glucosinolates-gene discovery and beyond. Trends Plant Sci 2010, 15(5):283.

7. Liu S, Kandoth PK, Warren SD, Yeckel G, Heinz R, Alden J, Yang C, Jamai A, El-Mellouki T, Juvale PS, et al: A soybean cyst nematode resistance gene points to a new mechanism of plant resistance to pathogens. Nature 2012, 492(7428):256. 
8. Hwang IS, An SH, Hwang BK: Pepper asparagine synthetase 1 (CaAS1) is required for plant nitrogen assimilation and defense responses to microbial pathogens. Plant J 2011, 67(5):749-762.

9. Van Damme M, Zeilmaker T, Elberse J, Andel A, de Sain-van der Velden M, van den Ackerveken G: Downy mildew resistance in Arabidopsis by mutation of HOMOSERINE KINASE. Plant Cell 2009, 21(7):2179.

10. Huibers RP, Loonen AEHM, Gao D, Van den Ackerveken G, Visser RGF, Bai Y: Powdery mildew resistance in tomato by impairment of SIPMR4 and SIDMR1. PLOS ONE 2013, 8:e67467.

11. Stuttmann J, Hubberten H-M, Rietz S, Kaur J, Muskett P, Guerois R, Bednarek P, Hoefgen R, Parker JE: Perturbation of Arabidopsis amino acid metabolism causes incompatibility with the adapted biotrophic pathogen Hyaloperonospora arabidopsidis. Plant Cell 2011, 23(7):2788-2803.

12. Liu G, Ji Y, Bhuiyan NH, Pilot G, Selvaraj G, Zou J, Wei Y: Amino acid homeostasis modulates salicylic acid-associated redox status and defense responses in Arabidopsis. Plant Cell 2010, 22(11):3845.

13. Whipps J, Budge S, Fenlon J: Characteristics and host range of tomato powdery mildew. Plant Pathology 1998, 47(1):36.

14. Bai $Y$, Huang C-C, van der Hulst R, Meijer-Dekens F, Bonnema G, Lindhout P: QTLs for tomato powdery mildew resistance (Oidium lycopersici) in Lycopersicon parviflorum G1. 1601 co-localize with two qualitative powdery mildew resistance genes. Mol Plant Microbe Interact 2003, 16(2):169-176.

15. Bai $Y$, van der Hulst R, Bonnema G, Marcel TC, Meijer-Dekens F, Niks RE, Lindhout P: Tomato Defense to Oidium neolycopersici: Dominant OI Genes Confer Isolate-Dependent Resistance Via a Different Mechanism Than Recessive ol-2. Mol Plant Microbe Interact 2005, 18(4):354-362.

16. Lindhout P, Beek Van Der H, Pet G: Wild Lycopersicon species as sources for resistance to powdery mildew (Oidium lycopersicum): mapping of the resistance gene $\mathrm{Ol}-1$ on chromosome 6 of L. hirsutum. Acta Horticulturae (ISHS) 1994, 376:387-394.

17. Li C, Bonnema G, Che D, Dong L, Lindhout P, Visser R, Bai Y: Biochemical and molecular mechanisms involved in monogenic resistance responses to tomato powdery mildew. Mol Plant Microbe Interact 2007, 20(9):1161.

18. Huang C-C, Hoefs Van De Putte PM, GHaanstra Van Der Meer J, MeijerDekens F, Lindhout P: Characterization and mapping of resistance to Oidium lycopersicum in two Lycopersicon hirsutum accessions: evidence for close linkage of two Ol-genes on chromosome 6 of tomato. Heredity 2000, 85(6):511-520.

19. Seifi A: Characterization of tomato genes for resistance to Oidium neolycopersici. PhD thesis. The Netherlands: Plant Breeding Department, Wageningen University; 2011

20. Seifi A, Kaloshian I, Vossen J, Che D, Bhattarai KK, Fan J, Naher Z, Goverse A Tjallingii WF, Lindhout P: Linked, if not the same, Mi-1 homologues confer resistance to tomato powdery mildew and root-knot nematodes. Mol Plant Microbe Interact 2011, 24(4):441-450.

21. Li C, Bai Y, Jacobsen E, Visser R, Lindhout P, Bonnema G: Tomato defense to the powdery mildew fungus: differences in expression of genes in susceptible, monogenic-and polygenic resistance responses are mainly in timing. Plant Mol Biol 2006, 62(1-2):127.

22. Pei D, Ma H, Zhang Y, Ma Y, Wang W, Geng H, Wu J, Li C: Virus-induced gene silencing of a putative glutathione $\mathrm{S}$-transferase gene compromised Ol-1-mediated resistance against powdery mildew in tomato. Plant Mol Biol Rep 2011, 29(4):972-978.

23. Duggleby RG, Pang SS: Acetohydroxyacid synthase. J Biochem Mol Biol 2000, 33(1):1.

24. McCourt J, Duggleby R: Acetohydroxyacid synthase and its role in the biosynthetic pathway for branched-chain amino acids. Amino Acids 2006, 31(2):173.

25. Duggleby RG, McCourt JA, Guddat LW: Structure and mechanism of inhibition of plant acetohydroxyacid synthase. Plant Physiol Biochem 2008, 46(3):309.

26. Tomato Functional Genomics Database. http://ted.bti.cornell.edu/cgi-bin/ TFGD/digital/home.cgi.

27. Binder S: Branched-chain amino acid metabolism in Arabidopsis thaliana. The Arabidopsis Book 2010:e0137.

28. Chen H, Saksa K, Zhao F, Qiu J, Xiong L: Genetic analysis of pathway regulation for enhancing branched-chain amino acid biosynthesis in plants. Plant J 2010, 63(4):573.

29. Chaleff RS, Bascomb NF, Chaleff RS, Bascomb NF: Genetic and biochemical evidence for multiple forms of acetolactate synthase in Nicotiana tabacum. Mol Gen Genet 1987, 210(1):33-38.
30. Scheel D, Casida JE: Sulfonylurea herbicides: Growth inhibition in soybean cell suspension cultures and in bacteria correlated with block in biosynthesis of valine, leucine, or isoleucine. Pestic Biochem Physiol 1985, 23(3):398.

31. Ray TB: Site of action of chlorsulfuron inhibition of valine and isoleucine biosynthesis in plants. Plant Physiol 1984, 75(3):827.

32. Royuela M, Arrese-Igor C, Muñoz-Rueda A, Gonzalez-Murua C: In vitro and in vivo effects of chlorsulfuron in sensitive and tolerant plants. J Plant Physiol 1991, 139(2):235.

33. Höfgen R, Laber B, Schüttke I, Klonus A-K, Streber W, Pohlenz H-D: Repression of acetolactate synthase activity through antisense inhibition (Molecular and biochemical analysis of transgenic potato (Solanum tuberosum L. cv Desiree) plants). Plant Physio/ 1995, 107(2):469-477.

34. Fakhoury AM, Lightfoot DA: Methods of using plants containing the gdhA gene. Patent 2013:US8383887.

35. Nolte $S$, Young B, Mungur R, Lightfoot D: The glutamate dehydrogenase gene gdhA increased the resistance of tobacco to glufosinate. Weed Res 2004, 44(4):335-339.

36. Ameziane R, Bernhard K, Lightfoot D: Expression of the bacterial gdhA gene encoding a NADPH glutamate dehydrogenase in tobacco affects plant growth and development. Plant and Soil 2000, 221(1):47-57.

37. Mungur R, Glass A, Goodenow D, Lightfoot DA: Metabolite fingerprinting in tranic Nicotiana tabacum altered by the Escherichia coli glutamate dehydrogenase gene. Journal of Biomedicine and Biotechnology 2005, 2005(2):198-214.

38. Zhao J, Williams CC, Last RL: Induction of Arabidopsis tryptophan pathway enzymes and camalexin by amino acid starvation, oxidative stress, and an abiotic elicitor. Plant Cell 1998, 10(3):359.

39. Song JT, Lu H, Greenberg JT: Divergent roles in Arabidopsis thaliana development and defense of two homologous genes, aberrant growth and death2 and AGD2-LIKE DEFENSE RESPONSE PROTEIN1, encoding novel aminotransferases. Plant Cell 2004, 16(2):353.

40. Sembdner G, Atzorn R, Schneider G: Plant hormone conjugation. Plan Mol Biol 1994, 26(5):1459-1481.

41. Fonseca S, Chini A, Hamberg M, Adie B, Porzel A, Kramell R, Miersch O, Wasternack C, Solano R: (+)-7-iso-Jasmonoyl-L-isoleucine is the endogenous bioactive jasmonate. Nat Chem Biol 2009, 5(5):344-350.

42. Park J-E, Park J-Y, Kim Y-S, Staswick PE, Jeon J, Yun J, Kim S-Y, Kim J, Lee Y-H, Park C-M: GH3-mediated auxin homeostasis links growth regulation with stress adaptation response in Arabidopsis. J Biol Chem 2007, 282(13):10036.

43. Liu Y, Schiff M, Dinesh-Kumar S: Virus-induced gene silencing in tomato. Plant J 2002, 31(6):777.

44. Helliwell CA, Wesley SV, Wielopolska AJ, Waterhouse PM: High-throughput vectors for efficient gene silencing in plants. Funct Plant Biol 2002, 29(10):1217.

45. Hurst CD, Knight A, Bruce IJ: PCR detection of genetically modified soya and maize in foodstuffs. Mol Breed 1999, 5(6):579.

46. Løvdal T, Lillo C: Reference gene selection for quantitative real-time PCR normalization in tomato subjected to nitrogen, cold, and light stress. Anal Biochem 2009, 387(2):238.

47. Livak KJ, Schmittgen TD: Analysis of Relative Gene Expression Data Using Real-Time Quantitative PCR and the $2^{-\Delta \Delta C T}$ Method. Methods 2001 25(4):402-408.

48. Pallant J: SPSS survival manual: A step by step guide to data analysis using SPSS (4 ${ }^{\text {th }}$ edition). Australia: Allen \& Unwin; 2011.

doi:10.1186/1471-2229-14-32

Cite this article as: Gao et al:: Down-regulation of acetolactate synthase compromises $\mathrm{Ol}-1$ - mediated resistance to powdery mildew in tomato. BMC Plant Biology 2014 14:32. 